prompts to discover their values. The guide provides HCPs structures and wording to address the following identified ACP topics: the identity of the child, living with illness, the future, hope, fears and worries, preferences for daily life and goals of care. The training educates HCPs about the concept of ACP and coping with illness and loss and trains specific communication skills. The intervention includes a documentation format.

Conclusion A pediatric ACP-intervention was designed targeted to the following needs: education about the concept of ACP, strategies to conduct ACP conversations and a documentation format. Our ongoing research will evaluate the feasibility of the intervention.

\section{P66 PEDIATRICIAN'S EXPERIENCES WITH ADVANCE CARE PLANNING: A QUALITATIVE STUDY}

${ }^{1} \mathrm{~J}$ Fahner*, ${ }^{1} \mathrm{~K}$ Prinsze, ${ }^{2} \mathrm{~J}$ Rietjens, ${ }^{2} \mathrm{~A}$ van der Heide, ${ }^{1} \mathrm{~J}$ van Delden, ${ }^{1} \mathrm{M}$ Kars. ${ }^{1}$ University Medical Center Utrecht, Utrecht, Netherlands; ${ }^{2}$ Erasmus MC, Rotterdam, Netherlands

10.1136/spcare-2019-ACPICONGRESSABS.147

Background Pediatricians in academic care centers are involved in high-complex care for children with life-limiting conditions. They guide families in defining goals of care and medical decision-making. Advance Care Planning (ACP) supports family-centered care and shared decision-making. This study evaluates how pediatricians integrate ACP in their daily care for children with life-limiting conditions.

Methods An interpretative qualitative study using thematic analysis was performed. Single interviews were undertaken with 17 pediatricians working in five pediatric tertiary hospitals.

Results Initiation of ACP discussions is determined by expected disease progression, technical treatment options or increasing symptoms. Pediatricians prefer to discuss ACP within a long-term relationship with a child's family. This ensures and enables them to address issues concerning challenging medical decisions and end-of-life in the right way at the right time for a specific family. Pediatricians focus in ACP discussions on future scenario's and related care options. They try to balance between 'hope' (normalizing living with illness, focus on problem solving) and 'the worst' (decline of the child's condition and an inevitable death). They aim to prepare the child and family for 'a life as normal a possible and when inevitable, a good end of life'. Few pediatricians explore the child's and family's values and perspectives.

Conclusion Pediatricians focus on prognosis and treatment options in ACP conversations. They take families by the hand throughout the disease trajectories and try to guide medical decision making in the best interest of the child. Exploration and integration of family values seems less integrated in their practice.

\section{P67 TESTING THE EFFECTIVENESS OF A FAMILY-CENTERED PEDIATRIC ADVANCE CARE PLANNING INTERVENTION: STUDY PROTOCOL FOR A RANDOMIZED CONTROLLED TRIAL}

A van Driessche* ${ }^{*}$ A de Vleminck, K Beernaert, J Cohen, L Deliens. VUB Brussel, BRUSSEL, Belgium

10.1136/spcare-2019-ACPICONGRESSABS. 148
Background A family-centered pediatric ACP intervention (FACE) was developed in the US, which demonstrated feasibility, acceptability and safety. The aim of this study is to evaluate the effectiveness of an ACP intervention, adapted from the FACE intervention, for pediatric oncology in Flanders, Belgium.

Methods A total of 93 dyads of parents and adolescents (age 10 - 18) receiving care in pediatric oncology wards will be recruited. Intervention dyads $(\mathrm{N}=46)$ will receive three weekly 60-minute sessions. Control dyads $(\mathrm{N}=46)$ will receive care as usual. Primary outcome is congruence in treatment preferences between adolescent and parent, measured by the Statement of Treatment Preference. Secondary outcomes are: quality of communication, decisional conflict for adolescents, and quality of life of adolescents and parents. Outcomes will be measured at baseline (T0) and 3 months after the intervention (T1). A process evaluation will be done by documentation of recruitment, analyzing audio recordings of the intervention, and post-trial qualitative interviews with adolescents $(n=10)$ and parents $(n=10)$ and focus groups with the involved healthcare professionals.

Discussion This will be the first pACP intervention in Europe and will provide evidence on the effectiveness of an ACP intervention in adolescents with cancer. A process evaluation will provide in-depth insight into how the pACP intervention was delivered in practice and contribute to understanding the underlying mechanisms of the intervention.

\section{P68 DEVELOPING A PEDIATRIC ACP INTERVENTION FOR ADOLESCENTS WITH CANCER IN BELGIUM}

A van Driessche* ${ }^{*}$ A de Vleminck, K Beernaert, J Cohen, L Deliens. VUB Brussel, Brussel, Belgium

\subsection{6/spcare-2019-ACPICONGRESSABS. 149}

Background Parents of adolescents with a life-limiting illness have expressed the desire to talk to their children about goals and preferences of care. Recently, a family-centered pediatric advance care planning (ACP) intervention (FACE) was developed in the US, which demonstrated feasibility, acceptability and safety. The aim of this project is to develop a pediatric ACP (pACP) intervention by translating the existing FACE intervention and adapting it to the Belgian situation for paediatric patients with cancer and their parents.

Methods First, feedback about the proposed content and process of the FACE intervention will be obtained from pediatricians $(n=4)$, psychologists $(n=4)$, parents and adolescents with cancer $(n=8)$ from four different pediatric oncology wards through semi-structured interviews. Second, four separate focus groups with the abovementioned groups will be organized. Third, experts with extensive experience in ACP with children will review the materials individually and findings will be discussed in an expert panel. Before pilot testing the intervention, cognitive testing of all questionnaires used for the outcome measurements with adolescents, their parent $(n=4)$ and physicians $(n=4)$ will be done. All retrieved information will be processed in a first version of a manual of how the intervention is delivered.

Discussion After development of the pACP intervention, effectiveness will be tested in a randomized controlled trial design. Primary objective of the trial is to assess whether the pACP intervention improves congruence in treatment preferences 
between paediatric patients and their parent. A process evaluation will provide more information about the underlying mechanisms of the intervention.

\section{P69 DEVELOPMENT AND EVALUATION OF AN ACP-PROGRAM FOR PROFESSIONALS IN PALLIATIVE CARE FOR PEOPLE WITH INTELLECTUAL DISABILITIES}

${ }^{1} \mathrm{AGFM}$ Vogel ${ }^{*},{ }^{1,2,3} \mathrm{AMA}$ Wagemans, ${ }^{4} \mathrm{H}$ Voss, ${ }^{4,5,6} \mathrm{AL}$ Francke, ${ }^{3} \mathrm{JFM}$ Metsemakers, ${ }^{2} \mathrm{AM}$ Courtens, ${ }^{4} \mathrm{AJE}$ de Veer. 'Maasveld, Koraal, Maastricht, Netherlands; ${ }^{2}$ Expertise Centre for Palliative Care, Maastricht University Medical Centre, Maastricht, the Netherlands; ${ }^{3}$ Department of Family Medicine, Faculty of Health, Medicine and Life Science, Maastricht University, the Netherlands; ${ }^{4}$ Netherlands Institute of Health Services Research (NIVEL), Utrecht, the Netherlands; ${ }^{5}$ Amsterdam UMC, VU Amsterdam, Amsterdam Public Health research institute, the Netherlands; ${ }^{6}$ Expertise Center for Palliative Care Amsterdam, Amsterdam UMC, location VU, Amsterdam, the Netherlands

\subsection{6/spcare-2019-ACPICONGRESSABS. 150}

Background People with intellectual disabilities (ID) have restricted cognitive and communicative abilities, and therefore encounter difficulties in clarifying their wishes and needs. Professionals in ID-care do not always recognize palliative care needs and lack communication skills to talk about future care in advance. Aim of this study was to develop and evaluate an advance care planning (ACP)-program to train professionals in knowledge and skills about ACP in palliative care for people with ID.

Methods This study contained five phases. Phase 1 consisted of a systematic review, (medical)file analysis and supplementary interviews, and depth-interviews to discover important aspects of ACP. These were translated into an ACP-program in co-creation with professionals, relatives and people with ID (phase 2). A communication training framework was developed (phase 3). Implementation of the ACP-program took place in six organizations providing ID-care (phase 4). In phase 5 the program was evaluated by pre-structured questionnaires send to participants of the program.

Results The ACP-program consists of 1.Methodology about important aspects of ACP, 2.ACP communication training, and 3.Consultation about implementation strategies for ACP. It is based on 10 competencies needed for ACP; palliative phase identification, signaling symptoms, communicating, documenting, structurally deploying ACP, taking wishes of people with ID into account, collaborating, paying attention to possible dilemmas, reflecting, and regarding $\mathrm{ACP}$ as a standard aspect of palliative care.

Conclusion An ACP program is helpful to make professionals aware of the importance of ACP and improve communication skills. Research is needed to further investigate the effective elements of the ACP program.

\section{P70 EFFECTS OF AN ADVANCE CARE PLANNING EDUCATIONAL PROGRAM INTERVENTION IN AN ACUTE HOSPITAL; A QUALITATIVE DATA ANALYSIS}

${ }^{1} \mathrm{M}$ Hamayoshi* ${ }^{2} \mathrm{~S}$ Goto, ${ }^{3} \mathrm{~A}$ Kono, ${ }^{1} \mathrm{C}$ Matsuoka, ${ }^{4} \mathrm{M}$ Ikenaga. ${ }^{1}$ Bukkyo University, Kyoto, Japan; ${ }^{2}$ Konan womans university, Kobe City, Japan; ${ }^{3}$ Osaka city university, Osaka, Japan; ${ }^{4}$ Yodogawa Christian hospital, Osaka, Japan

10.1136/spcare-2019-ACPICONGRESSABS. 151

Background Advance care planning (ACP) is a crucial end-oflife care practice. However, an ACP educational program for practitioners in an acute care setting has not yet been established. This study aimed to examine the effects of an ACP educational program in acute hospital. Aim: This work is evaluating the effect of ACP education programme on acute hospital practitioners. Design: A mixed-methods, Pre-and post test study to evaluate changing practitioner attitudes post program. The intervention program was three times during the 3 month in 90 minutes per session. As a program evaluation, comments on participant's questionnaires two questions about an attitude and practice were categorized and evaluated. We used conventional content analysis. Setting/Participants: The final sample of 63 practitioners whose they were working at B acute hospital.

Results As a result of analysis of qualitative data, Question1 was categorized into five categories, and Question2 was categorized into seven categories. The results that participants thought important of ACP implementation and talk with patient and family. It was suggested that participants were able to look back on their own way of thinking about death and found the tips of ACP implementation.

Conclusion The key message of this study was change sustained of awareness about positive attitude of ACP and EOL after 6 month intervention. These results suggest that the present ACP educational program was effective at improving staff attitudes towards the end of life care of patients.

\section{P72 TRANSLATION INTO SPANISH, CROSS-CULTURAL ADAPTATION AND VALIDATION OF AN ADVANCE CARE PLANNING SELF-EFFICACY SCALE: PRELIMINARY RESULTS}

${ }^{1} \mathrm{C}$ Lasmarias*, ${ }^{1} \mathrm{~A}$ Aradilla-Herrero, ${ }^{2} \mathrm{M}$ Subirana-Casacuberta, ${ }^{2} \mathrm{~S}$ Ela, ${ }^{3} \mathrm{~S}$ Delgado, ${ }^{4} X$ Gómez-Batiste. ${ }^{1}$ Chair of Palliative Care, Barcelona, El Salvador; ${ }^{2}$ Consorci Hospitalari de Vic, Vic, Spain; ${ }^{3}$ St Luke's Hospice, London, UK; ${ }^{4}$ University of Vic, Vic, Spain

\subsection{6/spcare-2019-ACPICONGRESSABS.152}

Background Advance Care Planning (ACP) explores patients' values and preferences to plan their care. Barriers for healthcare professionals to start it include fear to talk about end of life or lack of communication skills. Self-efficacy (SE) is a key factor that impacts learning about ACP. Measuring SE would show the impact of training to acquire ACP competencies. The ACP SE scale (ACP_SEs) of 17 items was validated in 2017; there aren't similar validated tools in Spanish.

Methods The ACP SEs was forward-backward translated. 10 ACP local experts tested it for clarity and comprehensibility. Validation: we designed a survey with 4 dimensions: sociodemographic variables; knowledge and self-perception on ACP; type of patients attended; 4 scales to predictive validity: ACP_SEs (Baughman, 2017); Trait Meta-Mood_s 24 (Salovey and Mayer, 1995); Personal Competence_s (Wallston, 1992); Coping with Death_s (Bugen, 1980). Participants: 5,500 professionals from 4 scientific societies: Palliative and Primary Care (3 societies); Geriatrics/Gerontologic (1society). Reliability will be determined by intraclass correlation coefficients, the measurement will be compared by T'Student and internal consistency by Cronbach's $\alpha$. Test-retest reliability will be quantified with a 4-week interval.

Results After the translation/adaptation process, the ACP_SEs has 19 items. New variables include to involve patients in $\overline{A C P}$ and to register ACP adequately. In a pilot with 47 professionals, consistency was $\alpha=0.909$. The results will confirm the scale's validity and show how professionals rate the ACP process. 